\title{
Unusual life-threatening Rosai-Dorfman disease of the trachea: role of NF-KB
}

\author{
Lin-Fu Zhou, ${ }^{1}$ Liang Chen, ${ }^{2}$ Quan Zhu, ${ }^{2}$ Cong Wang, ${ }^{3}$ Hai Xu ${ }^{4}$ Xue-Fan Cui, ${ }^{1}$ \\ Lei-Fu Jiang, ${ }^{1}$ Shao-Heng He, ${ }^{1}$ Mao Huang, ${ }^{1}$ Kai-Sheng Yin ${ }^{1}$
}

- An additional figure is published online only. To view this file please visit the journa online (http://thorax.bmj.com).

${ }^{1}$ Department of Respiratory Medicine, The First Affiliated Hospital, Nanjing Medical University, Nanjing, Jiangsu, China

${ }^{2}$ Department of Cardio-Thoracic Surgery, The First Affiliated Hospital, Nanjing Medical University, Nanjing, Jiangsu, China

${ }^{3}$ Department of Pathology, The First Affiliated Hospital, Nanjing Medical University, Nanjing,

Jiangsu, China

${ }^{4}$ Department of Radiology, The First Affiliated Hospital, Nanjing Medical University, Nanjing,

Jiangsu, China

\section{Correspondence to}

Dr Lin-Fu Zhou, Department of Respiratory Medicine, The First Affiliated Hospital, Nanjing Medical University, 300 Guangzhou Road, Nanjing, Jiangsu 210029, China;

Ifzhou@njmu.edu.cn

LC and $\mathrm{OZ}$ contributed equally to this article.

Received 4 April 2010 Accepted 29 July 2010

\section{ABSTRACT}

Rosai-Dorfman disease (RDD) is a rare non-neoplastic histioproliferative disorder characterised by painless lymphadenopathy, low fever, high erythrocyte sedimentation rate, leucocytosis and hypergammaglobulinaemia. Overactivity of nuclear factor $\kappa \mathrm{B}(\mathrm{NF}-\kappa \mathrm{B})$ is linked with inflammatory, cancerous and autoimmune diseases. The first case is described of an unusual life-threatening RDD of the trachea with no lymphadenopathy at risk of suffocation in a 39-year-old Chinese woman. A diagnosis of RDD was made following CT scans, thoracotomy and histological examination. Gel shift assay revealed an essential role for NF- $\mathrm{KB}$ overactivity in RDD. The patient remains well with no evidence of progression without treatment. Histological confirmation should be sought in all cases as the clinical manifestation of RDD is similar to asthma or lung carcinoma.

Sinus histiocytosis with massive lymphadenopathy (SHML) is a syndrome first recognised by Rosai and Dorfman in $1969 .{ }^{1}$ As a rare non-neoplastic histiocytic proliferative disorder, Rosai-Dorfman disease (RDD) is characterised by bilateral painless cervical lymphadenopathy as well as low fever, high erythrocyte sedimentation rate, leucocytosis and hypergammaglobulinaemia. Extranodal involvement including the skin, orbit and nasopharynx occurs in approximately one-third of patients. ${ }^{2}$ Overactivity of nuclear factor $\kappa \mathrm{B}(\mathrm{NF}-\kappa \mathrm{B}, \mathrm{p} 50 / \mathrm{p} 65)$ is linked with inflammatory, cancerous and autoimmune diseases. ${ }^{3}$ We describe an uncommon case of life-threatening RDD of the trachea in association with NF-KB overactivity.

\section{CASE REPORT}

A 39-year-old non-smoking Chinese woman presented with 1 month of dyspnoea. Her chest x-ray was normal and pulmonary function tests showed a severe obstructive pattern (ratio of forced expiratory volume in $1 \mathrm{~s}\left(\mathrm{FEV}_{1}\right)$ to forced vital capacity (FVC) $35 \%$ and $\mathrm{FEV}_{1} 39 \%$ predicted) with a plateau of the expiratory loop (figure 1A). She was unresponsive to 2 weeks of empirical steroid and bronchodilator therapy. She had been well at rest but became increasingly dyspneic on exertion and was admitted as an emergency. She had no chest pain, haemoptysis, weight loss or night sweats and denied sickle cell disease. Her history included hypertension, uterine fibroids and breast fibroma.

On presentation to the department she was in moderate distress with a respiratory rate of 25-30 breaths/min. Her physical examination showed mild three depression sign and low breath sounds without palpable lymphadenopathy. The erythrocyte sedimentation rate was high at $31 \mathrm{~mm} / \mathrm{h}$ (normal $20 \mathrm{~mm} / \mathrm{h}$ ). Arterial blood gases showed normal values at rest, whereas hypoxia and hypercapnia were found after exercise (pH 7.27 (normal 7.40), arterial oxygen tension $71 \mathrm{~mm} \mathrm{Hg}$ (normal $100 \mathrm{~mm} \mathrm{Hg}$ ), and arterial carbon dioxide tension $54 \mathrm{~mm} \mathrm{Hg}$ (normal $40 \mathrm{~mm} \mathrm{Hg}$ )) on room air. Other laboratory investigations, including $C$ reactive protein, complete blood count, renal and liver function tests, D-dimer, brain natriuretic peptide, tuberculin test, tumour markers, antinuclear antibody, rheumatoid factor, antineutrophil cytoplasmic and perinuclear antibodies, were all within normal limits. A bronchial dilation test was negative and $24 \mathrm{~h}$ peak expiratory flow rate was $10 \%$ (normal 20\%). Fiberoptic laryngoscopy revealed no circumferential subglottic narrowing or neoplasm. CT scans of the neck, chest and abdomen on day 2 demonstrated an ill-defined irregular soft tissue mass $1.5 \mathrm{~cm} \times 1.4 \mathrm{~cm}$ on cross section with a length of $2.7 \mathrm{~cm}$ in the trachea, $3.3 \mathrm{~cm}$ above the carina (figure 1B). Three-dimensional reconstruction and virtual bronchoscopy (figure 1C) confirmed about $86 \%$ obstruction of the intrathoracic trachea.

After multidisciplinary team discussion a decision was made not to carry out fine needle aspiration cytology or interventional therapy and a tracheal ring sleeve resection and reanastomosis was performed to prevent asphyxia on day 3 (see figure 1 in online supplement). A pale yellow mass was seen originating from and encasing the right wall of the trachea and invading into the tracheal adventitia. There was a $0.9 \mathrm{~cm} \times 0.8 \mathrm{~cm}$ paratracheal lymph node at station 2 . Given the benign nature of the rapid frozen sections illustrating non-malignant histiocytes in the mass and inflammatory cells in the lymph node, both were then removed and the thorax closed. Postoperative histological examination of the lymph node showed chronic inflammation with marked hyperplasia of histiocytes. Periodic acid-Schiff and acidfast stains were negative. Histological analysis of the mass showed the characteristic sinuses occupied by lymphocytes, plasma cells and histiocytes engulfing numerous intact lymphocytes within their eosinophilic cytoplasm, designated 'emperipolesis' (figure 1D). Immunohistochemistry showed that the histiocytes were reactive to $\mathrm{S} 100$ protein (figure 1E) and CD68 (figure 1F) but not CD1a, confirming a diagnosis of RDD. The patient remained well without any treatment or evidence of progression when last seen in July 2010. 
Figure 1 (A) Pulmonary function test showing severe airway obstruction with a plateau of the expiratory loop. $(B, C)$ Coronal reconstruction of chest CT scan and virtual bronchoscopy showing an irregular mass with $86 \%$ occlusion of the trachea.

(D) Histological examination revealed sinus histiocytosis with massive lymphadenopathy and emperipolesis (arrow). Magnification $\times 400$. $(\mathrm{E}, \mathrm{F})$ Immunohistochemistry demonstrating S100 protein and CD68positive. Magnification $\times 100$ and $\times 200$, respectively.
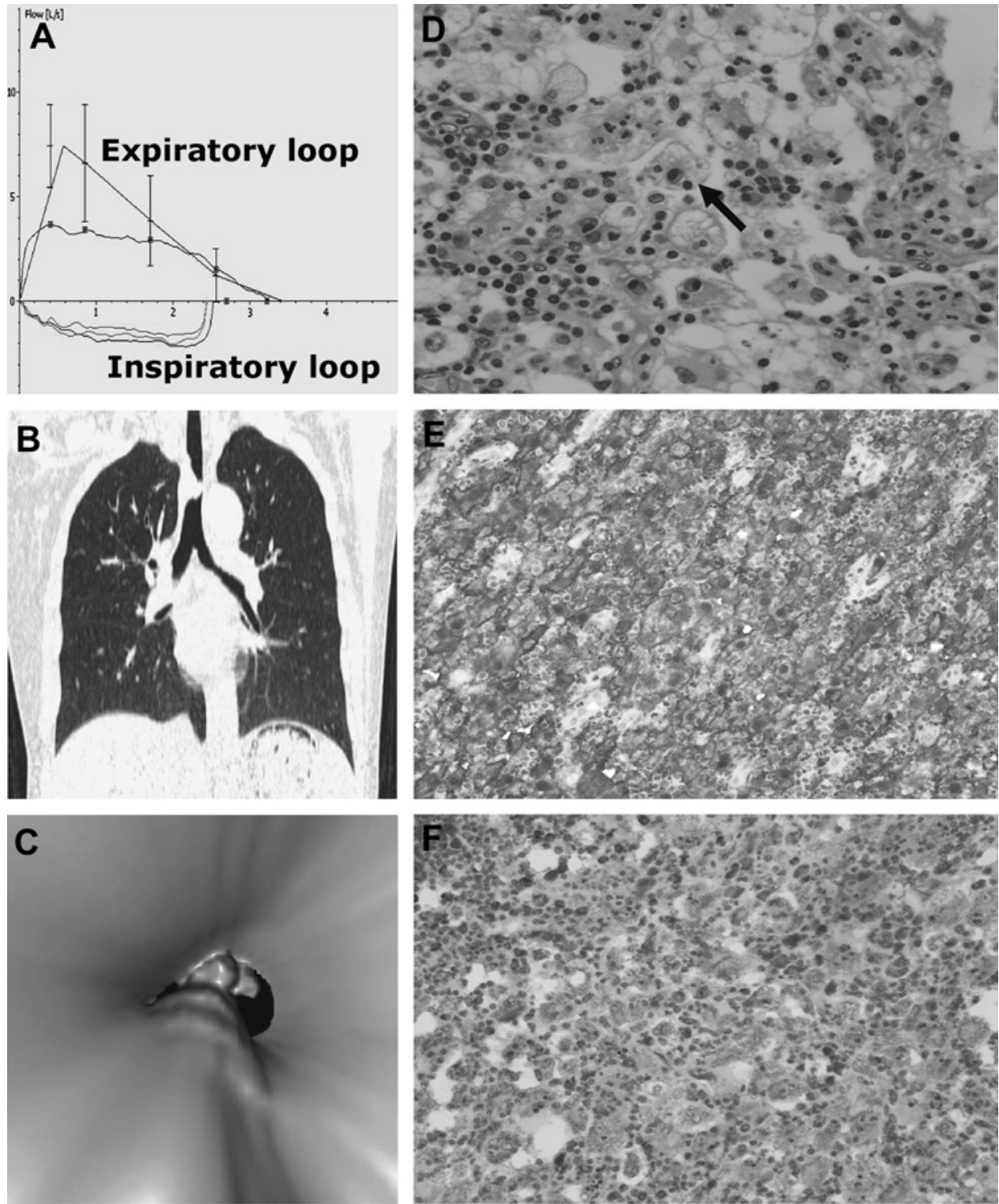

\section{Gel shift assay}

Nuclear proteins were extracted and gel shift assay was conducted as described previously. ${ }^{3}$ Apparently increased NF- $\mathrm{BB}$ activity was detected in the mass relative to that in the normal trachea (figure 2), demonstrating a potential role for $N F-\kappa B$ in the pathogenesis of RDD.

\section{DISCUSSION}

Confirmation of RDD is made by identification of emperipolesis and immunohistochemical staining with S100 protein+/CD68 $+/$ CD1a-. SHML may closely simulate other disease states in its clinical manifestations, especially malignant lymphoma, histiocytosis and non-neoplastic disorders including storage diseases. RDD is occasionally complicated by sickle cell disease, autoimmune lymphoproliferative syndrome and histiocytic sarcoma in the literature. ${ }^{45}$

RDD remains an enigmatic self-limiting disease, irrespective of an association with viruses, lymphoma, amyloidosis and other lymphoproliferative disorders. The treatment in most instances requires only observation due to spontaneous regression. Patients do not respond to antibiotics and the response to corticosteroids, radiation and chemotherapy is

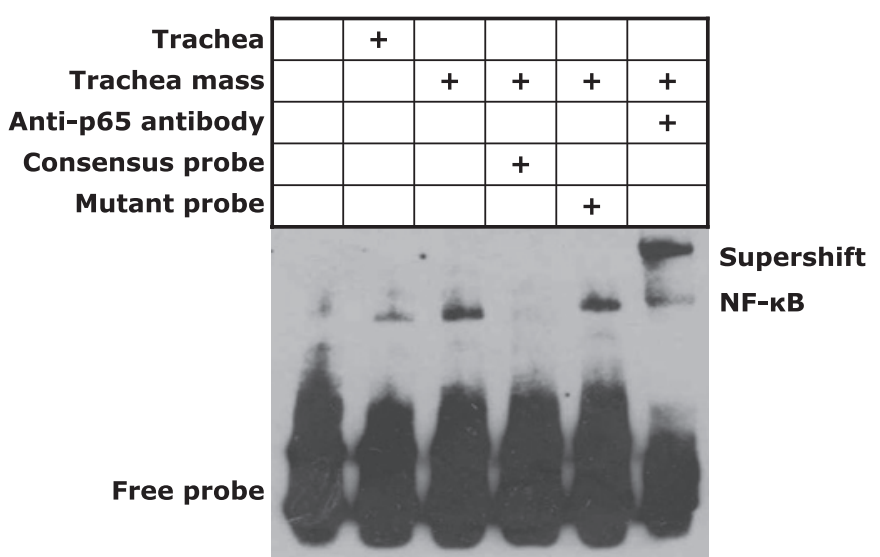

Figure 2 Gel shift assay showing overactivity of nuclear factor $\kappa B$ (NF- $\kappa B$ ) in a specimen of tracheal Rosai-Dorfman disease (RDD). The competition and supershift assays documenting the binding specificity and $\mathrm{p} 65$ subunit of NF-KB. 
inconsistent. In cases with significant cosmetic deformity or when there is fatal or functional obstruction, surgery is commonly indicated. ${ }^{6}$

Seven cases of non-lethal RDD involvement of the lower airway have been reported to date, including one case in an AfroCaribbean subject and two cases each in American, Caucasian and Chinese populations, mostly concomitant with lymphadenopathy. ${ }^{2-8}$ A female predominance (4:3) is found from 6 to 60 years of age. Thoractomy/tracheostomy is necessary in cases with severe trachea or upper airway obstruction.

To our knowledge, this is the first report of a patient with unusual life-threatening RDD of the trachea with no lymphadenopathy who was at risk of suffocation. We also speculate an essential role for NF- $\kappa \mathrm{B}$ overactivity in RDD. Its clinical manifestation is similar to asthma or lung carcinoma, so histological confirmation should be sought in all cases. ${ }^{8}$ It usually tends to regress without any intervention and has a good outcome, given the benign nature of RDD.

Acknowledgements The authors thank Dr Jijun Xu (Cleveland Clinic, Ohio, USA) for helpful comments and Professor Bruce S Bochner (Johns Hopkins Asthma and Allergy Center, Baltimore, Maryland, USA) for critical review of the manuscript.

Funding This work was supported by grants from the National Natural Science Foundation of China 30400191 and 30570797, Summit Project of Jiangsu Personnel 06B035, Jiangsu Key Principal Investigator of Medicine RC2007043, Science and
Technology Program for Returned Overseas Scholars 200886 and FD09 and Nanjing Science and Technology Project 200901078 (to L-FZ).

Competing interests None.

Patient consent Obtained.

Ethics approval This study was conducted with the approval of the Jiangsu Province Hospital ethics committee.

Contributors L-FZ diagnosed the patient and drafted the manuscript.

Provenance and peer review Not commissioned; externally peer reviewed.

\section{REFERENCES}

1. Rosai J, Dorfman RF. Sinus histiocytosis with massive lymphadenopathy: a newly recognized benign clinicopathological entity. Arch Pathol 1969;87:63-70.

2. Ottaviano G, Doro D, Marioni G, et al. Extranodal Rosai-Dorfman disease: involvement of eye, nose and trachea. Acta Otolaryngol 2006;126:657-60.

3. Zhou LF, Zhang MS, Hu AH, et al. Selective blockade of NF-KB by novel mutated I $\mathrm{KB} \alpha$ suppresses $\mathrm{CD} 3 / \mathrm{CD} 28$-induced activation of memory $\mathrm{CD}^{+}{ }^{+} \mathrm{T}$ cells in asthma. Allergy 2008;63:509-17.

4. Stebbing C, van der Walt J, Ramadan G, et al. Rosai-Dorfman disease: a previously unreported association with sickle cell disease. BMC Clin Pathol 2007:7:3.

5. Venkataraman G, McClain KL, Pittaluga S, et al. Development of disseminated histiocytic sarcoma in a patient with autoimmune lymphoproliferative syndrome and associated Rosai-Dorfman disease. Am J Surg Pathol 2010;34:589-94.

6. Carpenter RJ 3rd, Banks PM, McDonald TJ, et al. Sinus histiocytosis with massive lymphadenopathy (Rosai-Dorfman disease): report of a case with respiratory tract involvement. Laryngoscope 1978;88:1963-9.

7. Shi SS, Sun YT, Guo L. Rosai-Dorfman disease of the lung: a case report and review of the literatures. Chin Med J 2009:122:873-4.

8. Ali A, Mackay D. Rosai-Dorfman disease of the lung. Thorax 2009;64:908-9. 